\title{
Efeito do concentrado no desempenho produtivo, reprodutivo e econômico de vacas da raça Holandesa em pastagem de coast-cross
}

\author{
[Effect of the concentrate on the productive, reproductive and economic performances \\ of Holstein cows on coast-cross pastures] \\ D. Vilela ${ }^{1}$, A.M. Ferreira ${ }^{1}$, J.C. Resende ${ }^{1}$, J.A. Lima ${ }^{2}$, R.S. Verneque ${ }^{1}$ \\ ${ }^{1}$ Embrapa Gado de Leite \\ Rua Eugênio do Nascimento, 610 - Bairro Dom Bosco \\ 36038-330 - Juiz de Fora, MG \\ ${ }^{2}$ Instituto de Zootecnia - Nova Odessa, SP
}

\begin{abstract}
RESUMO
Avaliou-se o desempenho produtivo, reprodutivo e econômico de vacas da raça Holandesa, mantidas em pastagem de capim-coast-cross e suplementadas com 3 ou $6 \mathrm{~kg}$ de concentrado/vaca/dia. O pastejo foi sob lotação rotacionada, com um dia de ocupação dos piquetes e 25 ou 35 dias de descanso nas épocas de chuvas ou de seca, respectivamente. A produção de leite foi influenciada pela quantidade de suplemento fornecida. Vacas que receberam $6 \mathrm{~kg}$ de suplemento produziram mais leite $(19,1 \mathrm{~kg} / \mathrm{dia})$ do que as que receberam $3 \mathrm{~kg}(15,5 \mathrm{~kg} / \mathrm{dia})$. O primeiro estro pós-parto ocorreu aos 99,9 e 67,8 dias, respectivamente, pelos métodos de identificação visual e dosagem de progesterona, para vacas suplementadas com $3 \mathrm{~kg}$ de concentrado, e 72,9 e 54,5 dias para as suplementadas com $6 \mathrm{~kg}$; os intervalos de partos, obtidos na mesma ordem dos tratamentos citados, foram de 444,8 e 436,8 dias. O método visual para identificação de estros não foi preciso e comprometeu a rentabilidade da atividade.
\end{abstract}

Palavras-chave: bovino, produção de leite, desempenho reprodutivo, Cynodon, rentabilidade, suplementação

\begin{abstract}
Productive, reproductive and economic performance of Holstein cows raised on coast-cross pasture and supplemented either 3 or $6 \mathrm{~kg}$ of concentrate/cow/day were evaluated. Grazing was under rotating with one day of occupation of each feedlot and 25 or 35 days of resting in the rainy or dry seasons, respectively. Milk yield was higher for cows receiving $6 \mathrm{~kg}$ of concentrate $(19.1 \mathrm{~kg} /$ day) than for cows receiving $(15.5 \mathrm{~kg} /$ day). The first post-calving estrous occurred on 99.9 and 67.8 days, respectively, by visual and progesterone dosage methods for cows fed $3 \mathrm{~kg}$ of concentrate, and 72.9 and 54.5 days for those fed $6 \mathrm{~kg}$. The calving intervals were 444.8 and 436.8 days for cows fed $3 \mathrm{~kg}$ and $6 \mathrm{~kg}$ of concentrate, respectively. The visual method for estrous detection was not accurate and compromised business profitability.
\end{abstract}

Keywords: dairy cattle, milk yield, reproductive performance, profitability, supplementation

Recebido em 3 de janeiro de 2005

Aceito em 18 de dezembro de 2006

E-mail: sac@cnpgl.embrapa.br

Apoio: $\mathrm{CNPq}$ 


\section{INTRODUÇÃO}

A produção intensiva de leite em pasto tem sido a forma mais eficiente para se reduzir os custos e manter a competitividade e a sustentabilidade da exploração leiteira. Incrementar a produtividade tem sido o grande desafio da pesquisa, que, entre outros segmentos da investigação, busca incessantemente descobrir espécies forrageiras tropicais que, manejadas de forma correta, apresentem bom potencial para implementar lucro e competitividade na atividade.

As gramíneas do gênero Cynodon, em razão de suas boas características nutricionais, potencial produtivo, resposta à fertilização, adaptação a diferentes ambientes e flexibilidade de uso, vêm sendo intensamente pesquisadas no Brasil. A Embrapa Gado de Leite vem desenvolvendo pesquisas desde a década passada (Vilela et al.,1996; Alvim et al., 1997; 1999) para definir o melhor manejo para esta gramínea, visando à sua utilização na produção intensiva de leite. Os resultados têm sido promissores, conseguindo-se lotações de até 7 vacas/ha, com produções diárias de leite superiores a $100 \mathrm{~kg} / \mathrm{ha}$ (Vilela et al., 1996).

Uma das grandes vantagens das forrageiras do gênero Cynodon é a sua capacidade de resposta à adoção de tecnologias, notadamente fertilização, irrigação e pastejo rotacionado. Quando utilizadas para produção de leite, podem induzir consideráveis reduções de custos e aumento nos lucros (Vilela et al., 1996; Fontanelli et al., 2000).

A eficiência reprodutiva é um dos principais indicadores a considerar quando se pretende elevar a rentabilidade na exploração leiteira. Prolongados períodos de anestro após o parto aumentam o intervalo de partos e comprometem o desempenho da atividade. Ganhos na eficiência reprodutiva resultam em incrementos diretos e proporcionais no resultado econômico da propriedade. Os principais fatores que influenciam o intervalo de partos estão ligados ao manejo sanitário e alimentar do rebanho. $\mathrm{O}$ déficit energético afeta a reprodução e dietas capazes de suprir as exigências fisiológicas e reprodutivas podem ser uma alternativa para evitar longos períodos de anestro. Um caminho para reduzir o intervalo de partos é encurtar o intervalo parto-primeiro estro, o que pode ser obtido com o animal parindo em boa condição corporal e mantendo ou perdendo pouco peso no início da lactação. Nesse sentido, métodos auxiliares de identificação do estro são indispensáveis para a inseminação no momento correto e o melhor aproveitamento do potencial reprodutivo das fêmeas.

Desenvolveu-se o presente estudo com o objetivo de avaliar o efeito do concentrado sobre o desempenho produtivo, reprodutivo e econômico de vacas da raça Holandesa em pastagem de capim-coast-cross, buscando estratégia que identifique o nível ótimo de produção.

\section{MATERIAL E MÉTODOS}

O experimento foi realizado no campo experimental de Coronel Pacheco da Embrapa Gado de Leite, e ocupou área de 7ha, formada com pastagem de coast-cross Cynodon dactylon cv. Essa gramínea está sendo utilizada em pastejo rotacionado, com cinco vacas/ha. Foram utilizadas 36 vacas da raça Holandesa, com média de peso vivo de $560 \mathrm{~kg}$ e potencial de produção de 6.000 a $6.500 \mathrm{~kg} /$ lactação, divididas em quatro grupos iguais, com duas repetições de área por tratamento. Foram utilizados nove animais por área e 18 por tratamento, que tiveram à disposição 36 piquetes com $1.944 \mathrm{~m}^{2}$ cada, divididos por cerca elétrica. $\mathrm{O}$ período de ocupação dos piquetes foi de um dia, e o descanso variou de 25 a 35 dias, de acordo com o regime de chuvas e temperaturas ao longo do ano. Na seca (outono-inverno) utilizou-se o maior período de descanso. $\mathrm{O}$ experimento foi realizado durante três anos consecutivos (outubro/2000 a outubro/2003), totalizando 108 lactações (36 animais por ano).

A pastagem foi adubada com $1.000 \mathrm{~kg} / \mathrm{ha} / \mathrm{ano}$ da fórmula 20-05-20 (N- $\left.\mathrm{P}_{2} \mathrm{O}_{5}-\mathrm{K}_{2} \mathrm{O}\right)$, distribuídos a lanço em seis aplicações anuais, sempre após a saída dos animais dos piquetes. Nos meses menos chuvosos e nos veranicos, a pastagem foi irrigada por aspersão, sendo a quantidade de água em cada irrigação estabelecida por meio da fórmula $\mathrm{LB}=(\mathrm{CC}-\phi)$ Pr.Da.10 / Ef, em que:

LB é a lâmina bruta de água a aplicar (mm); CC é a capacidade de campo (g de água/g de solo); $\phi$ é o teor de umidade do solo à tensão de -6 a - 
7atm (g de água/g de solo); Pr é a profundidade efetiva do sistema radicular $(\mathrm{cm})$; Da é a densidade aparente do solo $\left(\mathrm{g}\right.$ de solo $\left./ \mathrm{cm}^{3}\right)$; e Ef a eficiência do sistema de irrigação.

Antes da entrada dos animais nos piquetes, foram colhidas amostras da forragem para avaliação qualitativa do pasto. Utilizou-se quadrado de $1 \mathrm{~m}$ de lado, lançado ao acaso, três vezes em cada piquete. Nesta avaliação foi desconsiderada a vegetação com altura inferior a $8 \mathrm{~cm}$. Os teores de proteína bruta e de fibra em detergente neutro e a digestibilidade in vitro da matéria seca encontram-se na Tab. 1.
Um grupo de vacas recebeu $3 \mathrm{~kg} / \mathrm{dia}$ e o outro $6 \mathrm{~kg} / \mathrm{dia}$ de concentrado, formulado com milho integral moído (62\%), soja integral tostada (35\%), mistura mineral $(2 \%)$ e calcário calcítico (1\%), que continha $19,5 \%$ proteína bruta, $9,3 \%$ de proteína degradável no rúmen e $86 \%$ de nutrientes digestíveis totais na matéria seca. $\mathrm{O}$ concentrado foi fornecido durante as ordenhas, realizadas duas vezes ao dia (7 e 15h). Encerrada a ordenha, as vacas eram reconduzidas aos piquetes.

Tabela 1. Médias de teores de matéria seca (MS), proteína bruta (PB) e fibra em detergente neutro (FDN) e de digestibilidade in vitro da matéria seca (DIVMS) do capim-coast-cross (três anos de avaliação)

\begin{tabular}{|c|c|c|c|c|}
\hline \multirow{2}{*}{ Estação } & MS & PB & FDN & DIVMS \\
\hline & \multicolumn{4}{|c|}{$(\%$ da MS) } \\
\hline Outono-inverno (período seco) & 25,5 & 16,0 & 63,3 & 65,4 \\
\hline Primavera-verão (período chuvoso) & 24,0 & 18,0 & 65,8 & 65,8 \\
\hline
\end{tabular}

No período de seca (de maio a setembro) de 2003, ano em que a disponibilidade de forragem atingiu nível inferior a $4.000 \mathrm{~kg}$ de $\mathrm{MS} / \mathrm{ha}$, suplementou-se o pasto com $17 \mathrm{~kg} / \mathrm{vaca} / \mathrm{dia}$ de silagem de milho com 33\% de MS. Em 2001 e 2002, essa suplementação não foi necessária. Ao longo de todo o ciclo de produção, as vacas foram pesadas a cada 15 dias. O escore da condição corporal (ECC) foi estimado nos dias de pesagem com base em avaliações visuais e táteis, atribuindo-se valores que variaram de 1 a 5 (Wildman et al., 1982). A produção de leite foi registrada diariamente. A secagem das vacas foi feita aos 60 dias antes do parto ou antes disso, quando a produção diária era inferior a $4 \mathrm{~kg}$ por vaca. A produção de leite foi corrigida para 3,5\% de gordura pela equação proposta por Skalan et al. (1992): $\mathrm{PLC}=(0,432+0,1625$ x G) $\times \mathrm{Q}$ em que: PLC é a produção corrigida para $3,5 \%$ de gordura; $\mathrm{G}$ é o percentual de gordura original; e $\mathrm{Q}$ a produção não corrigida.

Pelo método visual, os estros foram identificados pela manhã e à tarde, por quatro observadores, sendo dois retireiros e dois técnicos. Não foi utilizado rufião para auxiliar nesta identificação. A partir de 15 dias depois do parto, amostras de sangue foram coletadas, uma vez por semana, até a confirmação da prenhez, com a finalidade de avaliar a progesterona, com a qual foi possível identificar estros não observados pelo método visual, e identificar os casos de morte embrionária.

As variáveis avaliadas foram dias para manifestação do primeiro estro após o parto, período de serviço, intervalo de partos e produção de leite.

A produção de leite foi analisada conforme delineamento em blocos ao acaso, com duas repetições de área e dois níveis (tratamentos) de concentrado ( 3 ou $6 \mathrm{~kg} / \mathrm{vaca} / \mathrm{dia}$ ). O critério para formação dos blocos foi época de parição. Os dados relativos aos parâmetros reprodutivos, em razão da uniformidade dos animais, foram coletados seguindo delineamento inteiramente ao acaso, cujo modelo matemático foi $\mathrm{Y}_{\mathrm{ij}}=\mathrm{m}+\mathrm{t}_{\mathrm{i}}+$ $\mathrm{e}_{\mathrm{ij}}$, em que:

$\mathrm{Y}_{\mathrm{ij}}=$ valor observado para a característica no animal $\mathrm{j}$ do tratamento $\mathrm{i}$;

$\mathrm{m}=$ constante inerente a toda observação;

$\mathrm{t}_{\mathrm{i}}=$ efeito do tratamento $\mathrm{i}(\mathrm{i}=3$ ou 6$)$;

$\mathrm{e}_{\mathrm{ij}}=$ efeito do erro aleatório.

A análise de variância obedeceu aos procedimentos sugeridos no pacote estatístico SAS (User's..., 1996).

A análise de viabilidade econômica entre os dois níveis de concentrados foi feita mediante a quantificação e a análise do resultado monetário do modelo: $\mathrm{Re}=\Delta \mathrm{p}+\Delta \mathrm{p}^{\prime}+\Delta \mathrm{f}+\Delta \mathrm{k}+\Delta \mathrm{k}^{\prime}-\Delta \mathrm{c}$ em 
que: Re é o resultado econômico, ou saldo entre os incrementos de receitas e as despesas decorrentes do aumento na quantidade de concentrado; $\Delta \mathrm{p}$ é valor da produção adicional de leite devido ao aumento direto de produtividade das vacas; $\Delta \mathrm{p}^{\prime}$ é o valor da produção adicional relativa de leite, decorrente da redução no intervalo entre partos do rebanho; $\Delta \mathrm{f}$ é o valor da produção adicional de fêmeas, considerando a redução no intervalo de partos do rebanho; $\Delta \mathrm{k}$ é o valor da redução relativa nos juros sobre o capital mobilizado em terra, devido ao incremento na produtividade da pastagem; $\Delta \mathrm{k}^{\prime}$ é o valor da redução relativa dos juros e da depreciação do capital investido (exceto em terra) devido ao aumento da produtividade relativa do rebanho pela redução no intervalo entre partos; e $\Delta \mathrm{c}$ é o valor do incremento direto nas despesas com concentrados ao passar de 3 para $6 \mathrm{~kg} / \mathrm{vaca} / \mathrm{dia}$. O aumento de 3 para $6 \mathrm{~kg} / \mathrm{vaca} /$ dia na quantidade de concentrado somente será viável economicamente se o valor monetário de Re for positivo.

\section{RESULTADOS E DISCUSSÃO}

As médias das produções de leite nos três períodos de avaliação e no período total estão na Tab. 2.

Independentemente do período avaliado, a produção de leite das vacas que receberam $6 \mathrm{~kg}$ de concentrado diário foi maior $(\mathrm{P}<0,01)$ do que a produção das que consumiram $3 \mathrm{~kg}$. Entre os dois níveis avaliados, cada quilograma adicional de concentrado resulta em aumento de $1,2 \mathrm{~kg}$ de leite diário na produção, quantidade semelhante à observada por Alvim et al. (1997), que, ao avaliarem vacas holandesas em pastagem de capim-coast-cross, durante duas lactações consecutivas, encontraram aumento médio de $1,0 \mathrm{~kg}$ de leite por quilograma extra de concentrado, ao passar de 3 para $6 \mathrm{~kg} / \mathrm{vaca} /$ dia.

Tabela 2. Média de produção de leite em três períodos de avaliação da lactação e no geral de vacas da raça Holandesa em pastagem de capim-coast-cross, de acordo com os tramentos (média de três anos)

\begin{tabular}{ccccc}
\hline Concentrado & \multicolumn{4}{c}{ Produção de leite $(\mathrm{kg} / \mathrm{vaca} / \mathrm{dia})^{1}$} \\
\cline { 2 - 5 }$(\mathrm{kg} / \mathrm{vaca} / \mathrm{dia})$ & $0 \mathrm{a} \mathrm{100} \mathrm{dias}$ & $101 \mathrm{a} 200$ dias & 201 a 330 dias & 0 a 330 dias \\
\hline 3 & $19,0 \mathrm{~B} \pm 0,60$ & $15,50 \mathrm{~B} \pm 0,49$ & $11,9 \mathrm{~B} \pm 0,53$ & $15,5 \mathrm{~B} \pm 0,38$ \\
6 & $22,2 \mathrm{~A} \pm 0,85$ & $19,2 \mathrm{~A} \pm 0,64$ & $16,2 \mathrm{~A} \pm 0,63$ & $19,1 \mathrm{~A} \pm 0,45$ \\
Média & $20,6 \pm 0,54$ & $17,4 \pm 0,44$ & $14,1 \pm 0,46$ & $17,3 \pm 0,23$ \\
\hline
\end{tabular}

Médias seguidas de letras distintas na coluna diferem entre si pelo teste $\mathrm{SNK}(\mathrm{P}<0,01)$. $\mathrm{CV}=22,9 \%$.

A maior produção das vacas que receberam $6 \mathrm{~kg}$ pode ter sido reflexo da qualidade da dieta. Nos primeiros 100 dias da lactação, as produções foram de 22,2 e $19,0 \mathrm{~kg} / \mathrm{vaca} / \mathrm{dia}$ com 6 e $3 \mathrm{~kg}$ de concentrado, respectivamente (Tab. 2). Segundo Holmes e Wilson (1990), cada unidade de matéria seca ingerida na forma de concentrado estimula a redução de 0,5 a 0,8 unidade no consumo de MS proveniente do volumoso. Segundo esses autores, isto é devido ao efeito da substituição de nutrientes, o que provavelmente aconteceu no presente trabalho.

Não houve diferença $(\mathrm{P}>0,05)$ no teor de gordura do leite entre os tratamentos $(3,61 \%$ e $3,54 \%)$. Contudo, houve diferença $(\mathrm{P}<0,01)$ na média de produção de leite corrigida para $3,5 \%$ de gordura do leite, $16,09 \pm 0,44$ e $19,50 \pm 0,50 \mathrm{~kg} / \mathrm{vaca} / \mathrm{dia}$, respectivamente, com 3 ou $6 \mathrm{~kg}$ de concentrado.

A persistência na lactação foi maior quando as vacas receberam $6 \mathrm{~kg}$ de concentrado em relação às que receberam $3 \mathrm{~kg}$ (redução de $0,27 \mathrm{e}$ de
$0,35 \mathrm{~kg}$ de leite $/ \mathrm{vaca} / \mathrm{dia}$, respectivamente), fato já observado por Alvim et al. (1997), quando forneceram maior quantidade de concentrado na fase inicial da lactação.

O pasto deve ser a principal fonte de nutrientes para o animal, mas é necessário que ele seja bem manejado e formado com espécies forrageiras de potencial nutricional e produtivo elevados. De maneira geral, a composição química dos pastos fertilizados apresenta desequilíbrio na relação energia:proteína, podendo causar a concentração excessiva de proteína fermentada no rúmen. Nesse caso, é necessário neutralizar o excesso de nitrogênio amoniacal presente no rúmen, o que pode ser feito por meio de suplementação estratégica com concentrados específicos. Neste estudo, a utilização de concentrado com energia mais elevada e proteína de baixa degradação no rúmen, como a soja integral tostada, possivelmente evitou esse problema. 
O potencial produtivo da pastagem de Cynodon foi também avaliado em outros experimentos por Vilela et al. (1996, 2002) e Alvim et al. (1997, 1999). Os registros mostram produção diária de leite de até $104 \mathrm{~kg} / \mathrm{ha}$, com lotação de 7,3vacas/ha, e produção individual diária de $20 \mathrm{~kg} / \mathrm{vaca}$. No presente estudo, a produção diária $(15,5$ e $19,1 \mathrm{~kg} /$ vaca ou 77,8 e $94,0 \mathrm{~kg} / \mathrm{ha})$, com lotação fixa de cinco vacas, é condizente com a produção relatada acima. As pequenas variações na produção por área e na taxa de lotação podem ser explicadas pelo fato de a quantidade de fertilização nitrogenada utilizada $(200 \mathrm{~kg} / \mathrm{ha})$ ter sido menor do que a relatada nos estudos anteriores $(360 \mathrm{~kg} / \mathrm{ha} / \mathrm{ano})$. Quanto à produção individual, a utilização dos mesmos animais experimentais durante as três lactações sucessivas pode ter contribuído para uma pequena queda na produção, visto que nos estudos anteriores houve reposição de animais ao final de cada período experimental.

Não houve diferença $(\mathrm{P}>0,05)$ na produção de leite entre os anos, como também entre estações do ano $(\mathrm{P}>0,05)$, fato explicado pela manutenção equilibrada da qualidade do pasto ao longo dos anos (Tab. 1), conseguida pelo manejo correto em termos de rotatividade no pastejo, fertilização e irrigação.

Observou-se diferença $(\mathrm{P}<0,05)$ no ECC entre os dois níveis de concentrado (Tab. 3), semelhantemente ao que aconteceu com o peso vivo das vacas. Conforme Lago et al. (2001), o ECC varia em função do método adotado na avaliação, com os valores mais altos sempre indicando vacas com melhor condição corporal. Segundo Ferguson et al. (1994), tanto o excesso como o baixo peso vivo das vacas ao parto provocam transtornos, ou seja, problemas metabólicos, redução de produção, menor taxa de concepção e dificuldades no momento da parição.
Edmonson et al. (1989) observaram que vacas muito gordas são mais propensas à cetose, pois consomem menos alimentos logo após o parto e mobilizam mais reservas corporais. Ferguson et al. (1994) sugeriram valores diferentes para o ECC e apresentaram tabela com valores que variaram de acordo com a fase de produção: de 2,50 a 3,25 no início da lactação; de 2,75 a 3,25 no meio da lactação e de 3,00 a 3,50 no final da lactação. No presente estudo, os ECC observados enquadram-se no intervalo sugerido para o início da lactação, porém estão abaixo dos valores sugeridos para o meio e para o final, quando se forneceram $3 \mathrm{~kg}$ de concentrado. As vacas mobilizam reservas corporais e perdem peso para suprir o balanço energético negativo, o que possivelmente não ocorreu neste estudo com o fornecimento de $6 \mathrm{~kg}$ de concentrado, indicando que o consumo de nutrientes foi suficiente para atender às exigências referentes à mantença, à produção e à reprodução. Com o fornecimento de $3 \mathrm{~kg}$ de concentrado, as vacas tenderam a recuperar o peso vivo e o escore corporal no final da lactação, porém em níveis inferiores ao das que receberam $6 \mathrm{~kg}$ de concentrado, evidenciando maior mobilização de reservas corporais naquele caso.

O longo período de anestro pós-parto é o principal problema reprodutivo na pecuária bovina (Vries e Veerkamp, 2000). Para Stevenson (1996), o ideal é o intervalo de partos máximo de 365 dias, o que exige período de serviço não acima de 85 dias. Para as vacas participantes desta pesquisa (em boas condições corporais por ocasião do parto), a qualidade do pasto de capim-coast-cross e as suplementações foram suficientes para estimular o primeiro estro antes dos 85 dias (Tab. 4), verificado pelo nível de progesterona.

Tabela 3. Escore da condição corporal (ECC) em três períodos de avaliação da lactação e no geral, de vacas da raça Holandesa em pastagem de capim-coast-cross de acordo com os tratamentos

\begin{tabular}{cllll}
\hline $\begin{array}{c}\text { Concentrado } \\
\text { (kg/vaca/dia) }\end{array}$ & \multicolumn{5}{c}{ ECC (escala de 1 a 5) } \\
\cline { 2 - 5 } & Início & Meio & Final & Geral \\
\hline 3 & $2,68 \mathrm{~B} \pm 0,39$ & $2,50 \mathrm{~B} \pm 0,35$ & $2,64 \mathrm{~B} \pm 0,33$ & $2,61 \mathrm{~B} \pm 0,05$ \\
6 & $2,89 \mathrm{~A} \pm 0,26$ & $2,98 \mathrm{~A} \pm 0,36$ & $2,97 \mathrm{~A} \pm 0,32$ & $2,95 \mathrm{~A} \pm 0,05$ \\
Média & $2,79 \pm 0,06$ & $2,74 \pm 0,06$ & $2,81 \pm 0,05$ & $2,78 \pm 0,02$ \\
\hline
\end{tabular}

Médias seguidas de letras distintas na coluna diferem entre si pelo teste SNK $(\mathrm{P}<0,01)$. CV $-11,6 \%$ 
As vacas, ao permanecerem no experimento por mais de uma lactação, tenderam a reduzir o intervalo parto-primeiro estro. $\mathrm{Na}$ primeira parição, este intervalo foi de 91 dias pela observação visual e de 68 dias pela análise de progesterona e, na segunda parição, 85 e 60 dias, respectivamente. Pela determinação de progesterona, verificou-se que $3 \mathrm{~kg}$ de concentrado/dia foram suficientes para se obter intervalo parto-primeiro estro dentro do limite desejável (inferior a 85 dias), contudo ligeiramente superior ao registrado com $6 \mathrm{~kg}$ de concentrado. A identificação visual superestimou o intervalo parto-primeiro estro. Na comparação dos 364 estros observados pelos dois métodos, verificou-se que $44 \%$ não foram identificados visualmente, o que mostra a deficiência desse método, que pode comprometer a eficiência reprodutiva do rebanho e a rentabilidade da atividade. A quantidade de $3 \mathrm{~kg}$ de concentrado não teve influência sobre o reinício da atividade ovariana lútea cíclica pós-parto. Por meio da análise de progesterona, foi possível identificar $30 \%$ de casos de morte embrionária, que ocorreram em embriões com média de idade de 38 dias nas vacas que receberam $6 \mathrm{~kg}$ de concentrado e de 40 dias nas que receberam $3 \mathrm{~kg}$.

Tabela 4. Intervalo parto-estro (dias) de vacas da raça Holandesa em pastagem de capim-coastcross, com estro identificado por observação visual ou pela análise de progesterona, de acordo com os tratamentos

\begin{tabular}{|c|c|c|}
\hline \multirow{2}{*}{$\begin{array}{l}\text { Concentrado } \\
\text { (kg/vaca/dia) }\end{array}$} & \multicolumn{2}{|c|}{$\begin{array}{l}\text { Intervalo parto-estro } \\
\text { (dias) }\end{array}$} \\
\hline & $\begin{array}{c}\text { Observação } \\
\text { visual }\end{array}$ & Progesterona \\
\hline 3 & $99,9 \mathrm{~A} \pm 8,13$ & $67,8 \mathrm{~A} \pm 4,80$ \\
\hline 6 & $72,9 \mathrm{~B} \pm 8,13$ & $54,5 \mathrm{~B} \pm 4,69$ \\
\hline Média & $86,4 \pm 5,75$ & 61,1 \\
\hline $\mathrm{CV}$ & $39,9 \%$ & $49,8 \%$ \\
\hline
\end{tabular}

Médias seguidas de letras distintas na coluna diferem entre si pelo teste SNK $(\mathrm{P}<0,05)$.

$\mathrm{O}$ intervalo de partos não foi diferente $(\mathrm{P}>0,05)$ entre as vacas que receberam 3 ou $6 \mathrm{~kg}$ de concentrado/dia (Tab. 5), provavelmente em razão da boa condição corporal ao parto, com os animais apresentando reserva corporal suficiente para atender à reprodução, em caso de possível balanço energético negativo no pós-parto. A média do intervalo de partos de 14,3 meses encontra-se nos limites preconizados para vacas com as características das utilizadas nesta pesquisa, bem abaixo da média (mais de 18 meses) verificada por Ferreira et al. (1997), em rebanhos de bacias leiteiras do estado do Rio de Janeiro.

Embora a média do intervalo parto-primeiro estro, verificado pela concentração de progesterona, tenha sido de apenas 61 dias, a média do intervalo de partos de 440,8 dias representa o período de serviço de 150 dias, ou seja, 85,5 dias a mais do que o primeiro estro verdadeiro pós-parto. Essa diferença entre o primeiro estro pós-parto e a concepção pode ser explicada por vários fatores, entre eles: alta taxa de cios não identificados pelo método visual (44\%), o que elevou a média do intervalo partoprimeiro estro para 86,4 dias; elevado índice de morte embrionária (30\%); e qualidade do sêmen, horário de inseminação e estresse calórico.

Tabela 5. Intervalo de partos (dias) de vacas da raça Holandesa em pastagem de capim-coastcross, de acordo com os tratamentos

\begin{tabular}{cc}
\hline $\begin{array}{c}\text { Concentrado } \\
\mathrm{kg} / \text { vaca/dia })\end{array}$ & $\begin{array}{c}\text { Intervalo de partos } \\
\text { (dias) }^{1}\end{array}$ \\
\hline 3 & $444,8 \mathrm{~A} \pm 17,16$ \\
6 & $436,8 \mathrm{~A} \pm 15,26$ \\
Média & $440,8 \pm 11,27$ \\
CV & $14,9 \%$
\end{tabular}

Médias seguidas de letras iguais na coluna não diferem pelo teste SNK $(\mathrm{P}>0,05)$.

Com o aumento do fornecimento de concentrado, alguns indicadores físicos de produção melhoraram: elevação de média da produção de leite por vaca (15,5 para 19,2 litros/dia); melhora na produtividade da terra $(77,8$ para 94,0 litros/ha/dia); diminuição de número de dias para manifestação do cio (99,9 dias para 72,9 dias); diminuição do número de dias do intervalo de partos (444,8 para 436,8 dias) e alteração na persistência da lactação $(0,35$ para $0,27 \mathrm{~kg}$ de leite/dia). Com esses resultados, além da produção adicional de leite, houve redução relativa no pagamento de juros sobre o capital imobilizado, redução no valor das depreciações, aumento na quantidade relativa de fêmeas nascidas ao longo do ano e redução no percentual de vacas secas na composição geral do rebanho. A lotação da pastagem foi mantida fixa em 5 vacas/ha, mesmo sabendo que $6 \mathrm{~kg}$ de concentrado permitiriam suportar maior carga 
animal do que a suplementação com $3 \mathrm{~kg}$ de concentrado. À luz desses resultados, quantificou-se monetariamente os elementos do modelo proposto para examinar a viabilidade de aumento do concentrado $(\operatorname{Re}=\Delta \mathrm{p}+\Delta \mathrm{p}+\Delta \mathrm{f}+$ $\left.\Delta \mathrm{k}+\Delta \mathrm{k}^{\prime}-\Delta \mathrm{c}\right)$. Os valores resultantes dos cálculos encontram-se na Tab. 6 .

$\mathrm{O}$ aumento de $3 \mathrm{~kg}$ diários de concentrado para $6 \mathrm{~kg}$ por vaca foi viável do ponto de vista econômico, pois incrementou a receita líquida da atividade em $\mathrm{R} \$ 1.839,10$ por ha. Esse valor foi calculado levando em conta a redução de 27 dias no intervalo de partos de 444,8 dias. Quando se efetuam esses mesmos cálculos, para o caso de uma redução de oito dias em 444,8 dias (Tab. 5), chega-se, ainda, ao aumento nas receitas líquidas de R\$ 1.151,95 por ha. Portanto, para a relação preço do leite:preço do concentrado vigente em Minas Gerais, em novembro de 2004, quantidades menores de concentrado ( $3 \mathrm{~kg} / \mathrm{vaca} / \mathrm{dia})$, para o caso específico de vacas de alta produção, resultariam em rentabilidade menor, considerando pastagem de boa qualidade e cinco vacas por hectare. A menor quantidade de concentrado passaria a ser viável somente na situação em que o preço do leite pago ao produtor permanecesse $15 \%$ a $20 \%$ menos em relação ao preço do concentrado. No presente estudo, o potencial de produção das vacas e, principalmente, a possibilidade de incrementar seus índices produtivos e reprodutivos, quando alimentadas com mais concentrado, foi decisivo no resultado econômico positivo alcançado.

Tabela 6. Saldo das receitas e despesas devido ao aumento da quantidade de concentrado de 3 para $6 \mathrm{~kg} / \mathrm{vaca} / \mathrm{dia}$ fornecido para vacas da raça Holandesa mantidas em pastagem de capim-coast-cross com lotação de 5 vacas/ha

\begin{tabular}{|c|c|c|}
\hline Variável & $\mathrm{R} \$$ /ha/5vacas/ano). & Observações \\
\hline$\Delta \mathrm{p}$ & $4.084,72$ & $\begin{array}{l}\text { Valor da produção adicional de leite, considerando o preço de R\$ } \\
0,62 \text { / litro (nov/ 2004, Minas Gerais) }\end{array}$ \\
\hline$\Delta \mathrm{p}^{\prime}$ & 916,23 & $\begin{array}{l}\text { Redução de } 27 \text { dias no intervalo de partos de } 444,83 \text { dias } \\
\text { (acréscimo de } 6,1 \% \text { no total de lactações encerradas em } 365 \text { dias). } \\
\text { Preço do leite: R\$ 0,62 / litro (nov/2004, Minas Gerais) }\end{array}$ \\
\hline$\Delta \mathrm{f}$ & 45,51 & $\begin{array}{l}\text { Redução de } 27 \text { dias no intervalo de partos de } 444,83 \text { dias } \\
\text { (acréscimo de } 6,1 \% \text { no total de partos ocorridos em } 365 \text { dias). } \\
\text { Preço das fêmeas: R\$ 300,00/animal (nov/2004, Minas Gerais) }\end{array}$ \\
\hline$\Delta \mathrm{k}$ & 62,47 & $\begin{array}{l}\text { Aumento de } 21 \% \text { na produtividade da terra; preço de } \mathrm{R} \$ \\
3.000,00 / \text { ha (nov/2004); taxa anual de juros de } 10 \%\end{array}$ \\
\hline$\Delta \mathrm{k}^{\prime}$ & 15,17 & $\begin{array}{l}\text { Redução de juros e depreciações do capital investido (exceto em } \\
\text { terra) decorrente do aumento de produtividade do rebanho (redução } \\
\text { do intervalo de partos) }\end{array}$ \\
\hline$\Delta \mathrm{c}$ & $3.285,00$ & $\begin{array}{l}\text { Despesas com o acréscimo de concentrado de } 3 \text { para } 6 \mathrm{~kg} / \mathrm{vaca} / \mathrm{dia} \text {. } \\
\text { Preço de } \mathrm{R} \$ 0,60 / \mathrm{kg} \text { de concentrado (nov/2004) }\end{array}$ \\
\hline $\mathrm{Re}$ & $1.839,10$ & $\begin{array}{l}\text { Margem bruta (valor total das receitas subtraído do valor total de } \\
\text { despesas) }\end{array}$ \\
\hline
\end{tabular}

\section{CONCLUSÕES}

A pastagem de capim-coast-cross de boa qualidade, adequadamente manejada, fertilizada, irrigada e suplementada, foi capaz de suprir as exigências nutricionais de vaca da raça Holandesa com produção diária de leite de até $19,1 \mathrm{~kg} /$ vaca, possibilitando taxa de lotação de 5 vacas/ha. O tempo para manifestação do estro depois do parto e o intervalo de partos no sistema de criação estudado evidenciaram que o retorno econômico da produção foi maior quando se forneceram $6 \mathrm{~kg}$ diários de concentrado por animal. Nesse sistema e com vacas holandesas de alto potencial de produção, recomenda-se a suplementação diária com $6 \mathrm{~kg}$ de concentrado por animal, por proporcionar maior produção de leite, melhor índice reprodutivo, maior persistência na lactação e maior lucratividade. 


\section{REFERÊNCIAS BIBLIOGRÁFICAS}

ALVIM, M.J.; VILELA, D.; LOPES, R.S. Efeito de dois níveis de concentrado sobre a produção de leite de vacas da raça Holandesa em pastagem de coast-cross. Rev .Soc. Bras. Zootec., v.26, p.967-975, 1997.

ALVIM, M.J.; VERNEQUE, R.S.; VILELA, D. et al. Estratégia de fornecimento de concentrado para vacas da raça Holandesa em pastagem de coast-cross. Pesqu. Agropec. Bras., v.34, p.1711-1720, 1999.

EDMONSON, A.J.; LEAN, I.J.; WEAVER, L.D. et al. A body condition scoring chat Holstein dairy cows. J. Dairy Sci., v.72, p.68-78, 1989.

FERGUSON, J.D.; BYERS, D.; FERRY, J. et al. Round table discussion: body condition of lactating cows. Agric. Pract., v.15, p.17-21, 1994.

FONTANELLI, R. S.; SOLLENBERGER, L.E.; STAPLES, C.R. Dairy cow performance pasture-based feeding systems and in confinement. Gainesville, Florida: University of Florida, Agronomy Department, 2000. 23p.

FERREIRA, A.M.; TEIXEIRA, S.R.; SANTOS, P.C. B. et al. Taxa de natalidade em rebanhos leiteiros do Estado do Rio de Janeiro. Rev. Bras. Reprod. Anim., v.21, p.122-124, 1997.

HOLMES, C. W.; WILSON, G. F. Produção de leite a pasto. Campinas: Instituto Campineiro de Ensino Agrícola, 1990. 708p.

LAGO, E. P.; PIRES, A. V.; SUSIN, I. et al. Efeito da condição corporal ao parto sobre alguns parâmetros do metabolismo energético, produção de leite e incidência de doenças no pósparto de vacas leiteiras. Rev. Bras. Zootec., v.30, p.1544-1549, 2001.

SKALAN, D.; ASHKENAZI, R.; BRAUN, A. et al. Fatty acids calcium soaps of fatty acids, and cottonseeds fed to high yielding cows. J. Dairy Sci., v.75, p.2463-2472, 1992.

USER'S guide: Stat version 6.11. Cary, NC: SAS Institute, 1996.

STEVENSON, J. Is there an optimal calving interval? Hoard's Dairyman, v.141, p.408, 1996.

VILELA, D.; ALVIM, M. J.; CAMPOS, O. F. et al. Produção de leite de vacas Holandesas em confinamento ou em pastagem de coast-cross. Rev. Soc. Bras. Zootec., v.25, p.1228-1244, 1996.

VILELA, D.; MATOS, L. L.; ALVIM, M. J. et al. Utilização de gordura protegida durante o terço inicial da lactação de vacas leiteiras, em pastagem de coast-cross. Pesqu. Agropecu. Bras., v.37, p.1503-1509, 2002.

VRIES, M.J.; VEERKAMP, R.F. Energy balance of dairy cattle in relation to milk production variables and fertility. J. Dairy Sci., v.83, p.62-69, 2000.

WILDMAN, E.E.; JONES, G.M.; WAGNER, P.E. et al. A dairy cow body condition scoring system and its relationship to selected productions characteristics. Anim. Prod., v.38, p.22-33, 1982. 